\title{
961.
}

\section{A TRIGONOMETRICAL IDENTITY.}

[From the Messenger of Mathematics, vol. xxiv. (1895), pp. 49-51.]

THE following was proposed as a Senate House Problem: Given the equations

$$
\begin{aligned}
& a \cos (\beta+\gamma)+b \cos (\beta-\gamma)+c=0, \\
& a \cos (\gamma+\alpha)+b \cos (\gamma-\alpha)+c=0, \\
& a \cos (\alpha+\beta)+b \cos (\alpha-\beta)+c=0,
\end{aligned}
$$

it is to be shown that $a^{2}+2 b c-b^{2}=0$.

Assume

$$
\cos \alpha+i \sin \alpha, \cos \beta+i \sin \beta, \cos \gamma+i \sin \gamma=x, y, z,
$$

then the equations are

$$
\begin{aligned}
& a\left(y z+\frac{1}{y z}\right)+b\left(\frac{y}{z}+\frac{z}{y}\right)+2 c=0 \\
& a\left(z x+\frac{1}{z x}\right)+b\left(\frac{z}{x}+\frac{x}{z}\right)+2 c=0 \\
& a\left(x y+\frac{1}{x y}\right)+b\left(\frac{x}{y}+\frac{y}{x}\right)+2 c=0
\end{aligned}
$$

that is,

$$
\begin{aligned}
& a\left(1+y^{2} z^{2}\right)+b\left(y^{2}+z^{2}\right)+2 c y z=0, \\
& a\left(1+z^{2} x^{2}\right)+b\left(z^{2}+x^{2}\right)+2 c z x=0, \\
& a\left(1+x^{2} y^{2}\right)+b\left(x^{2}+y^{2}\right)+2 c x y=0,
\end{aligned}
$$

the second and third equations give

$$
a: b: 2 c=x\left(x^{2}-y z\right): x\left(-1+x^{2} y z\right):\left(1-x^{4}\right)(y+z) ;
$$


or, say $a, b, 2 c$ are equal to these values; and then, substituting in the first equation, we have

which is

$$
x\left(1+y^{2} z^{2}\right)\left(x^{2}-y z\right)+x\left(y^{2}+z^{2}\right)\left(-1+x^{2} y z\right)+\left(1-x^{4}\right)\left(y^{2} z+y z^{2}\right)=0,
$$

$$
(x-y)(x-z)\{x+y+z-(y z+z x+x y)(x+y+z)\}=0 \text {, }
$$

viz. our relation between $x, y, z$ is

or, what is the same thing,

$$
x+y+z-(y z+z x+x y) x y z=0,
$$

Then

$$
\frac{1}{y z}+\frac{1}{z x}+\frac{1}{x y}-(y z+z x+x y)=0
$$

$$
\begin{gathered}
a+b=x\left(-1+x^{2}\right)(1+y z), \\
a-b=x\left(1+x^{2}\right)(1-y z), \\
2 c=\left(1-x^{4}\right)(y+z), \\
a^{2}-b^{2}=x^{2}\left(1-x^{4}\right)\left(y^{2} z^{2}-1\right), \quad 2 b c=x\left(-1+x^{2} y z\right)\left(1-x^{4}\right)(y+z) .
\end{gathered}
$$

The equation to be verified is

that is,

$$
x\left(y^{2} z^{2}-1\right)=\left(1-x^{2} y z\right)(y+z),
$$

as it should be.

$$
x+y+z-(y z+2 x+x y) x y z=0 \text {, }
$$

A somewhat different form of the proof is as follows:-We have identically

$$
\begin{aligned}
& \left|\begin{array}{ccc}
\cos (\beta+\gamma), & \cos (\beta-\gamma), & 1 \\
\cos (\gamma+\alpha), & \cos (\gamma-\alpha), & 1 \\
\cos (\alpha+\beta), & \cos (\alpha-\beta), & 1
\end{array}\right| \\
& =4 \sin \frac{1}{2}(\beta-\gamma) \sin \frac{1}{2}(\gamma-\alpha) \sin \frac{1}{2}(\alpha-\beta)\{\sin (\beta+\gamma)+\sin (\gamma+\alpha)+\sin (\alpha+\beta)\},
\end{aligned}
$$

and therefore the relation between the angles is

$$
\sin (\beta+\gamma)+\sin (\gamma+\alpha)+\sin (\alpha+\beta)=0 .
$$

From the second and third equations,

$$
a: b: c=\sin \left\{\frac{1}{2}(\beta+\gamma)-\alpha\right\}:-\sin \left\{\frac{1}{2}(\beta+\gamma)+\alpha\right\}: 2 \sin \alpha \cos \alpha \cos \frac{1}{2}(\beta-\gamma),
$$

or say

therefore

$$
\begin{aligned}
& a=\sin \frac{1}{2}(\beta+\gamma) \cos \alpha-\cos \frac{1}{2}(\beta+\gamma) \sin \alpha \\
& b=-\sin \frac{1}{2}(\beta+\gamma) \cos \alpha-\cos \frac{1}{2}(\beta+\gamma) \sin \alpha, \\
& c=2 \sin \alpha \cos \alpha \cos \frac{1}{2}(\beta-\gamma)
\end{aligned}
$$

$$
\begin{aligned}
a^{2}-b^{2} & =-4 \sin \alpha \cos \alpha \sin \frac{1}{2}(\beta+\gamma) \cos (\beta+\gamma)=-2 \sin \alpha \cos \alpha \sin (\beta+\gamma), \\
b c & =2 \sin \alpha \cos \alpha\left\{-\cos \frac{1}{2}(\beta-\gamma) \sin \frac{1}{2}(\beta+\gamma) \cos \alpha-\cos \frac{1}{2}(\beta-\gamma) \cos \frac{1}{2}(\beta+\gamma) \sin \alpha\right\}, \\
& =2 \cdot \frac{1}{2} \sin \alpha \cos \alpha\{-(\sin \beta+\sin \gamma) \cos \alpha-(\cos \beta+\cos \gamma) \sin \alpha\}, \\
& =\sin \alpha \cos \alpha\{-\sin (\gamma+\alpha)-\sin (\alpha+\beta)\}=\sin \alpha \cos \alpha \sin (\beta+\gamma),
\end{aligned}
$$


whence therefore

$$
a^{2}-b^{2}+2 b c=0
$$

which is the required relation.

The equation to be proved may also be written

$$
\begin{array}{lll}
\cos (\beta+\gamma), & \cos (\beta-\gamma), & 1 \\
\cos (\gamma+\alpha), & \cos (\gamma-\alpha), & 1 \\
\cos (\alpha+\beta), & \cos (\alpha-\beta), & 1
\end{array}
$$

$=4 \sin \frac{1}{2}(\beta-\gamma) \sin \frac{1}{2}(\gamma-\alpha) \sin \frac{1}{2}(\alpha-\beta)\{\sin (\beta+\gamma)+\sin (\gamma+\alpha)+\sin (\alpha+\beta)\}$,

or putting

$$
\begin{array}{ll}
\beta+\gamma=a, & b-c=\gamma-\beta \\
\gamma+\alpha=b, & c-a=\alpha-\gamma \\
\alpha+\beta=c, & a-b=\beta-\alpha
\end{array}
$$

this becomes

$$
\begin{aligned}
& \left|\begin{array}{ccc}
\cos a, & \cos (b-c), & 1 \\
\cos b, & \cos (c-a), & 1 \\
\cos c, & \cos (a-b), & 1
\end{array}\right| \\
& =-4 \sin \frac{1}{2}(b-c) \sin \frac{1}{2}(c-a) \sin \frac{1}{2}(a-b)(\sin a+\sin b+\sin c),
\end{aligned}
$$

an identity which may be proved without difficulty. 